\title{
Vertrauen durch Transparenz
}

\section{Beim Ranking der Nachhaltigkeitsberichte siegte die Firma Henkel knapp vor KarstadtQuelle und dem Otto-Versand. Die Ergebnisse geben Aufschlïsse wie Nachhaltigkeit von fortschrittlichen großen Unternehmen interpretiert wird und welche Branchen aktiv sind.}

B

Von Thomas Loew und Jens Clausen ereits zum fünften Mal haben das Institut für ökologische Wirtschaftsforschung (IÖW) und future e.V. die Qualität der deutschen Umweltund Nachhaltigkeitsberichte der größten deutschen Konzerne analysiert. In das Ranking wurden die Nachhaltigkeitsberichte der 150 größten deutschen Unternehmen aus Industrie, Dienstleistung, Handel, Banken und Versicherungen einbezogen. Von diesen Unternehmen lagen im Herbst 2004 genau 53 eigene Nachhaltigkeitsberichte vor. Bei weiteren 25 Konzernen lag ein Nachhaltigkeitsbericht des ausländischen Mutterkonzerns vor. Diese Berichte wurden jedoch nicht bewertet, weil der Fokus auf die Unternehmen mit deutschen Konzernzentralen gesetzt wurde. Eine negative Wertung der internationalen Berichte ist mit diesem Ausschluss nicht verbunden.

Weiterhin ist zu berücksichtigen, dass nur die Nachhaltigkeitsberichte sowie vergleichbare Umwelt oder CSR-Berichte und nicht das gesamte Informationsangebot des Unternehmens bewertet wurden. Neben den Inhalten des Berichts wurden nur solche Informationen berücksichtigt, auf die direkt verwiesen wird. Denn der ideale Bericht verschafft einen Gesamtüberblick, informiert zu den bei dem Unternehmen relevanten Nachhaltigkeitsaspekten und vermittelt interessierten Stakeholder einen schnellen Zugang zu den gewünschten Informationen.

\section{Bewertungsmethode}

Für die systematische und nachvollziehbare Bewertung der Berichte wurden unter Berücksichtigung zahlreicher Vorarbeiten detaillierte Anforderungen entwickelt (1). Diese Anforderungen sind in 13 Haupt- und 48 Unterkriterien gegliedert. Die ökologischen Anforderungen umfassen die ökologischen Aspekte der Produktion sowie der Produkte und Dienstleistungen. Im Vergleich zum Ranking 2000 wurden hier Umstellungen vorgenommen. Beispielsweise wurde Biodiver- sität in anbetracht der Bedeutung des Themas in der angloamerikanischen Berichtsdebatte aufgenommen. Energieverbrauch und Klimaschutz wurden deutlicher hervorgehoben.

Die Darstellung der sozialen Leistungen des Unternehmens wird mit den Kriterien Interessen der Mitarbeiter, soziale Verantwortung im Umfeld und soziale Aspekte der Produkte und Dienstleistungen geprüft. Die dort im Einzelnen definierten Anforderungen sind im Vergleich zum Ranking der Umweltberichte weitgehend neu. Dennoch haben sie sich bewährt, denn für fast alle Anforderungen liegen Best Practice Beispiele vor.

Ökologische und soziale Anforderungen sind in der Bewertungsmethode mit jeweils rund 40 Prozent gleich gewichtet. Die restlichen 20 Prozent der Gewichtung entfallen auf allgemeine Anforderungen wie Glaubwürdigkeit und Vergleichbarkeit. Insgesamt können bei der Bewertungsmethode 700 Ergebnispunkte erreicht werden. Die Methode und die genaue Bewertungsskala wurden im Januar von veröffentlicht.

\section{Gewinner}

Gewinner des Rankings 2005 ist der Nachhaltigkeitsbericht von Henkel mit 494 Punkten. Der Bericht zeichnet sich durch eine sehr gute Darstellung der Themen Produktsicherheit, Mitarbeiter- belange und Arbeitszeiten sowie die beste Erfuillung der integrierten Anforderungen aus. Auch hat Henkel eine gute Systematik für Querverweise auf seine Unternehmenswebsite entwickelt.

Den zweiten Platz nimmt der Bericht von KarstadtQuelle ein, dicht gefolgt von dem Bericht des Branchenwettbewerbers OTTO. Beide Berichte weisen hohes Niveau in der Darstellung ihrer Umweltschutz- und sozialen Aktivitäten auf. An Platz vier präsentiert der Axel Springer Verlag einen Bericht, der die Umweltanforderungen zu 79 Prozent und damit als Bester erfïllt.

Adidas-Salomon konnte mit einer ehrlichen Darstellung der sozialen Herausforderungen in der Lieferantenkette punkten. Der Nachhaltigkeitsbericht von Volkswagen wurde als bester Bericht aus der Automobilbranche bewertet und erreichte insgesamt Rang sieben. Unter den Top Ten befinden sich außerdem RWE, die HVB Group, die BMW Group und die Deutsche Telekom.

Der höchste Grad an professioneller Berichterstattung ist in den Branchen Automobilhersteller, Papier und Druck, Transport und Kommunikation sowie Chemie/ Pharma festzustellen. In diesen Branchen sind Nachhaltigkeitsberichte weit verbreitet, wobei die Automobilhersteller die Nase vorne haben. Bei den Autobauern berichten alle sechs Unternehmen und gleichzeitig ist die Qualität der Berichte mit einer durchschnittlichen Punktzahl von 359 beim Rankingdurchschnitt von 325 überdurchschnittlich hoch. Die Ausnahme ist Porsche: Das Unternehmen hat den mit großem Abstand schlechtesten Bericht seiner Branche veröffentlicht.

Den dritten Platz nimmt die Gruppe der Unternehmen aus den Branchen Transport und Kommunikationsdienstleistungen ein, in der vier Unternehmen zu den besten 35 gehören. Branchensieger ist die Deutsche Telekom. $\mathrm{Zu}$ den Schlusslichtern der Gruppe zählt die Thomas

\begin{tabular}{|ll|l|l|}
\hline \multicolumn{4}{|c|}{ Tabelle 1: Die Top 10 im Ranking der Nachhaltigkeitsberichte } \\
\hline 1 & Henkel KGaA & Nachhaltigkeitsbericht 2003 & 494 \\
\hline 2 & KarstadtQuelle AG & Besser miteinander - Nachhaltigkeitsbericht 2003 & 490 \\
\hline 3 & OTTO (GmbH \& Co KG) & Bewusstsein(s)formen - Nachhaltigkeitsbericht 2004 & 478 \\
\hline 4 & Axel Springer Verlag AG & Nachhaltigkeitsbericht 2003 & 462 \\
\hline 5 & Adidas-Salomon AG & 2003 & 454 \\
\hline 6 & RWE AG & Unsere Verantwortung - Bericht 2003 & 449 \\
\hline 7 & HVB Group & $\begin{array}{l}\text { Nachhaltigkeit bewegt 2002 (vom Umweltbericht zum } \\
\text { Nachhaltigkeitsbericht) }\end{array}$ & 448 \\
\hline 7 & Volkswagen AG & Umweltbericht 2003/2004 & 448 \\
\hline 9 & BMW Group & Sustainable Value Report 2003/2004 & 417 \\
\hline 10 & BSH - Bosch Siemens Hausgeräte GmbH & Verantwortung für Umwelt und Gesellschaft 2003/2004 & 412 \\
\hline 10 & Deutsche Telekom AG & Personal- und Nachhaltigkeitsbericht 2004 & 412 \\
\hline
\end{tabular}


Cook AG, auf deren Website im Bereich der Unternehmensdarstellung nicht der kleinste Hinweis auf Umwelt- oder Sozialverantwortung zu entdecken ist.

In der Branche Chemie/ Pharma berichten immerhin drei Viertel der Unternehmen, doch die Qualität der Berichte schneidet mit einer Punktzahl von 316 bei einem Gesamtschnitt von 325 nur durchschnittlich ab. In der Branche ist eine große Spreizung von sehr guten Berichten bei Henkel, BASF und Degussa bis hin zu sehr schwachen Berichten wie bei Braun Melsungen und Beiersdorf festzustellen.

Immerhin die Hälfte der Banken hat einen Bericht zu Umwelt oder Nachhaltigkeit veröffentlicht. Mit 323 Ergebnispunkten liegt die durchschnittlich Qualität der Berichterstattung im Mittelfeld. Den besten Bericht in der Branche hat die bayrische Hypovereinsbank vorgelegt. Sechs der größten 15 Institute, unter anderem die DZBank, Bankgesellschaft Berlin und HSH Nordbank, berichten in keiner Weise über ihre gesellschaftlichen Verantwortlichkeiten.

Die deutschen Versicherer sind dagegen sehr rückständig. Von den 17 größten Versicherungsunternehmen legen nur fünf überhaupt einen Bericht auf und die Berichte sind bis auf eine Ausnahme eher dürftig.

Die in den 90er Jahren in der Umweltberichterstattung gesammelte Erfahrung lässt sich an den Ergebnissen einfach ablesen: Die Umweltanforderungen wurden durchschnittlich zu 48 Prozent erfüllt, während die sozialen Anforderungen durchschnittlich nur zu einem Drittel realisiert wurden.

Bemerkenswerterweise ist der Erfüllungsgrad für alle drei Hauptkriterien dieses Bereichs nahezu identisch:

- Interessen der Mitarbeiter (36 Prozent)

- Soziale Verantwortung im Umfeld (32 Prozent)

- Soziale Aspekte der Produkte und Dienstleistungen (31 Prozent)

Genauer betrachtet, zeigt sich indessen, dass bei den zwei Kriterien „Interessen der Mitarbeiter“ (Qualifikation, Gleichstellung, Arbeitszeiten etc.) und „Soziale Verantwortung im Umfeld“ (Spenden, Antikorruption, Verbandsarbeit etc.) der beste Bericht jeweils 68 Prozent der erreichbaren Punkte erzielte. Demgegenüber kam bei den Anforderungen zu ,Soziale Aspekte der Produkte und Dienstleistungen" (Arbeitsbedingungen, (Supply chain etc.) kein Bericht auf mehr als 43 Prozent der erreichbaren Punkte. Dieses Thema harrt also noch einer adäquaten Darstellung.
In diesen Ergebnissen wird deutlich, dass die heutige Nachhaltigkeitsberichterstattung im Kern eine um das Soziale erweiterte Umweltberichterstattung ist. Obwohl mit der Sozialberichterstattung Erfahrungen seit den 70er Jahren vorliegen, scheint diese „Neuaufnahme“ nicht einfach und verlangt intensiv danach, neue und mehr Erfahrungen zu sammeln.

\section{Nichtberichterstatter}

Obwohl großen Unternehmen eine besondere Verantwortung gegenüber der Gesellschaft zugesprochen wird und sie diese auch für sich reklamieren wenn sie gegen Regulierungen argumentieren, berichten immer noch nicht alle ganz großen Unternehmen. Knapp die Hälfte der deutschen Konzerne erstellt keinen Nachhaltigkeitsbericht. Hierzu zählen die DAX 30 Unternehmen Commerzbank, Eon, Altana und Thyssen-Krupp. Und bei einem Viertel der Größten haben wir überhaupt keine öffentlichen Informationen zur Nachhaltigkeit entdecken können. $\mathrm{Zu}$ diesen „schwarzen Schafen“ des Rankings zählen die HUK-Coburg, die R\&V Versicherungen, Rheinmetall, Aldi, REWE und Schlecker. Wirklich intransparent sind also nicht die Unternehmen mit einem vielleicht noch lückenhaften und verbesserungswürdigen Nachhaltigkeitsbericht, sondern diejenigen, die entweder keinen Bericht zur gesellschaftlichen Verantwortung erstellen oder gar keinerlei Informationen herausgeben.

\section{Anmerkung}

(1) Loew, T./ Clausen, J.: Nachhaltigkeitsberichterstattung in Deutschland: Ergebnisse und Trends im Ranking 2005.

Berlin 2005, Download unter www.ranking-nachhaltigkeitsberichte.de.

\section{Die Autoren}

Thomas Loew ist Geschäftsführer des Institute 4 Sustainability. Dr. Jens Clausen ist wissenschaftlicher Mitarbeiter im Forschungsfeld ökologische Unternehmenspolitik am Institut für ökologische Wirtschaftsforschung (IÖW).

Kontakt: Institute 4 Sustainability. Tel: 030-4435

2094, E-Mail: Loew@4sustainability.org,

IÖW-Hannover, E-Mail: Jens.Clausen@ioew.de, Tel: $511-1640342$

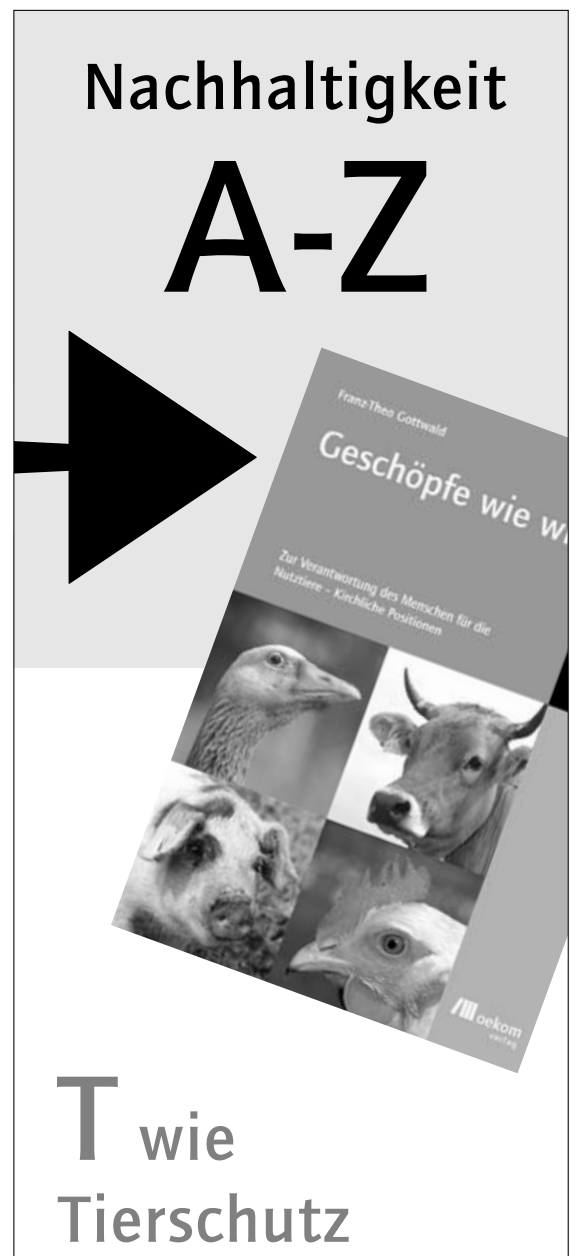

Die Position der Kirchen zur menschlichen Verantwortung für das Nutztier wird in diesem Buch umfassend vorgestellt, aber auch kritisch beleuchtet.

Franz-Theo Gottwald zeigt Perspektiven auf, um das Miteinander von Mensch, Tier und Natur zu verbessern und die Umwelt in ein nachhaltiges Gleichgewicht zu bringen. Neue Blickwinkel für Menschen, denen Tierschutz am Herzen liegt.

Franz Theo Gottwald

Geschöpfe wie wir

Zur Verantwortung des Menschen für die Nutztiere - Kirchliche Positionen München 2004; 133 Seiten; 9,80€ ISBN 3-936581-54-1

\section{Erhältlich bei}

www.oekom.de

oekom@wmi-verlagsservice.de

Fax ++49/(0)8191/125-405

\section{/ll oekom verlag}

Die guten Seiten der Zukunft 
(c) 20I0 Authors; licensee IÖW and oekom verlag. This is an article distributed under the terms of the Creative Commons Attribution Non-Commercial No Derivates License (http://creativecommons.org/licenses/by-nc-nd/3.o/), which permits unrestricted use, distribution, and reproduction in any medium, provided the original work is properly cited. 